\title{
BMJ Open Role of neutrophil-to-lymphocyte ratio as a prognostic biomarker in patients with breast cancer receiving neoadjuvant chemotherapy: a meta- analysis
}

\author{
Qiong Zhou (D) , ${ }^{1}$ Jie Dong, ${ }^{1}$ Qingqing Sun, ${ }^{1}$ Nannan $L u,{ }^{2}$ Yueyin Pan, ${ }^{2}$ \\ Xinghua $\mathrm{Han}^{2}$
}

To cite: Zhou Q, Dong J, Sun Q, et al. Role of neutrophilto-lymphocyte ratio as a prognostic biomarker in patients with breast cancer receiving neoadjuvant chemotherapy: a meta-analysis. BMJ Open 2021;11:e047957. doi:10.1136/ bmjopen-2020-047957

- Prepublication history and additional supplemental material for this paper are available online. To view these files, please visit the journal online (http://dx.doi.org/10.1136/ bmjopen-2020-047957).

Received 14 December 2020 Accepted 06 September 2021

A) Check for updates

(c) Author(s) (or their employer(s)) 2021. Re-use permitted under CC BY-NC. No commercial re-use. See rights and permissions. Published by BMJ.

${ }^{1}$ Department of Oncology, Provincial Hospital Affiliated to Anhui Medical University, Hefei, China

${ }^{2}$ Division of Life Sciences and Medicine, The First Affiliated Hospital of USTC, Hefei, Anhui, China

Correspondence to

Dr Xinghua Han;

hxhmail@ustc.edu.cn

\section{ABSTRACT}

Objective The neutrophil-to-lymphocyte ratio (NLR) is recognised as a suitable prognostic biomarker in patients with breast cancer. Nevertheless, the efficacy of this biomarker in predicting the pathological complete response (pCR) and survival in patients with breast cancer receiving neoadjuvant chemotherapy (NACT) is still controversial. This meta-analysis aimed to identify the association between baseline NLR and the prognosis of patients with breast cancer treated with NACT.

Design Meta-analysis.

Data sources Relevant literature published before 1 May 2021 was searched using the Cochrane Library, Embase, PubMed and the Web of Science databases.

Eligibility criteria All studies involving patients with breast cancer treated with NACT and peripheral blood pretreatment NLR recorded as a dichotomous variable were included.

Data extraction and synthesis Two researchers independently extracted and evaluated $\mathrm{OR} / \mathrm{HR}$ and its $95 \%$ Cls of survival outcomes and clinicopathological parameters.

Results A total of 19 studies were identified. From each study, the impact of NLR on the pCR, OR and HR, with their $95 \%$ Cls were extracted and combined using either a random or fixed-effects model. The results indicate that a higher pCR in patients with a low NLR (OR 1.620, 95\% Cl 1.209 to 2.169, $p<0.001$ ). In addition, an elevated NLR predicted lower disease-free survival (HR $2.269,95 \% \mathrm{Cl}$ 1.557 to 3.307, $p<0.001$ ) and overall survival (HR 1.691, $95 \% \mathrm{Cl} 1.365$ to $2.096, \mathrm{p}<0.001$ ) in patients with breast cancer treated with NACT.

Conclusions NLR is a suitable biomarker for predicting $\mathrm{pCR}$ and survival in patients with breast cancer receiving NACT.

\section{INTRODUCTION}

Breast cancer has the highest incidence and cancer-related mortality in women worldwide. ${ }^{1}$ Neoadjuvant chemotherapy (NACT) is often used to downstage the tumour to facilitate breast-conserving surgery in patients
Strengths and limitations of this study

- This is the first meta-analysis to assess the role of neutrophil-to-lymphocyte ratio (NLR) as an immunity/inflammation biomarker in predicting pathological complete response and survival in patients receiving neoadjuvant chemotherapy (NACT).

- This study provides preliminary evidence for NLR to predict the treatment response and prognosis of patients with breast cancer with NACT.

- Scientific and reliable statistical methods were applied.

- The results of this study could provide a strategy for further large-sample prospectively randomised controlled studies.

- All the studies included in our analysis were retrospective and lacked detailed information on the tumour subtypes, potentially biasing the results of our research findings.

who might have needed a mastectomy at initial diagnosis. Additionally, NACT has an important role in assessing the efficacy of chemotherapy early on during the treatment, hence reducing the risk of providing ineffective toxic therapy. ${ }^{2}$ However, studies have shown that pathological complete response (pCR) was more likely to occur in a subgroup of patients with breast cancer treated with NACT, eventually leading to improved survival when compared with patients who still had residual disease after surgery. ${ }^{3}$ Numerous genetic mutations, including TP53, PIK3CA, CDKN2A and SIRT5, have been associated with a worse response to NACT in breast cancer. ${ }^{4}$ However, these mutations are difficult to trace, and until now, no single genetic marker has been found to be clinically suitable to predict the tumour response after NACT. Therefore, there is a need to identify 
better prognostic serum biomarkers to optimise the treatment for patients with breast cancer.

Recent studies have found an association between tumour progression and systemic inflammatory response to treatment. ${ }^{5-7}$ Various inflammatory markers have been identified as prognostic markers for different types of cancers. The neutrophil-to-lymphocyte ratio (NLR) is one of the most commonly used markers and is calculated through dividing the number of neutrophils by the number of lymphocytes. ${ }^{8-11}$ NLR has been linked with poor survival in several cancers, including gastrointestinal and ovarian cancers. ${ }^{12}$ Furthermore, some studies found that a higher NLR may lead to a worse response to NACT and worse survival in different types of solid tumours. ${ }^{13}$ Yet, the role of NLR as a prognostic biomarker for patients with breast cancer treated with NACT is still not clear, highlighting the need for a meta-analysis to assess the role of NLR as a biomarker in predicting pCR and survival in these patients.

Therefore, this meta-analysis aimed to evaluate the relationship between pretreatment NLR, pCR, and survival in patients with breast cancer treated with NACT.

\section{MATERIAL AND METHODS \\ Search strategy}

A systematic literature search of all articles published up to 1 May 2021 was conducted based on the following databases: Embase, PubMed, The Cochrane Library and Web of Science databases. The keywords used for the search strategy included variations of the term "NLR" (eg, "NLR, "neutrophil/lymphocyte", "neutrophil-tolymphocyte", "neutrophil to lymphocyte ratio", "neutrophil" and "lymphocyte"), "Breast cancer" (eg, "Breast cancer", "breast carcinoma", "malignant breast tumor", "mammary cancer," "breast neoplasms") and "neoadjuvant chemotherapy" (eg, "neoadjuvant chemotherapy", "preoperative systemic treatment", "primary chemotherapy", "preoperative chemotherapy", "pre-surgical treatment"). The reference list of the retrieved articles was also reviewed to identify relevant articles.

\section{Inclusion and exclusion criteria}

pCR was defined as the absence of residual cancer in both breast tissue and nodes following surgery. All studies involving patients with breast cancer who were treated with NACT and had the peripheral blood pretreatment NLR recorded as a dichotomous variable were included. Furthermore, only the studies that had complete treatment outcomes reported in the form of either pCR, breast cancer-specific and/or disease-free survival (DFS), overall survival (OS), HR and OR with their 95\% CIs, were included.

Abstracts, case studies, letters, reviews, nonclinical studies and studies not written in English were excluded. Studies were also excluded if they had repeated data or had insufficient data to estimate the HR or OR with 95\% CI, NLR presented as a continuous variable or a non-binary variable, and missing information on the number of patients achieving pCR in the low and high NLR groups.

\section{Data extraction and quality control}

Two researchers independently extracted and evaluated relevant data from the research articles or abstracts. If the two researchers did not reach an agreement, a third researcher was consulted. The year of publication, first author and location were extracted for each study. Furthermore, the total number of cases in the pCR and non-pCR groups, age, follow-ups, tumour subtype and stage, the technique used to calculate NLR, and NLR thresholds were recorded. The treatment outcomes, including neoadjuvant treatment response, survival outcome information, and HRs with 95\% CIs, were subsequently extracted. The researchers used the NewcastleOttawa Scale (NOS) rating scale to assess the quality of the included studies. ${ }^{14}$ This technique involves assigning a quality rating to each study using a scale ranging from zero to nine, based on three broad group categories, including the comparability of the groups, the selection of the study groups, and the outcome of the exposure. The higher the NOS score, the higher the quality of the study.

\section{Patient and public involvement}

No patient involved.

\section{Statistical analysis}

The pCR rate, ORs, HRs and 95\% CIs for the high and low-NLR groups were either extracted from the study or calculated using the data available. ${ }^{15}$ A OR greater than 1 indicates that a low NLR is associated with improved pCR rate, while an HR greater than 1 shows poorer outcomes in patients with breast cancer with high NLR expression. HR estimates were weighted and pooled using the generic inverse-variance and random-effects model according to Cochrane Handbook for Systematic Reviews of Interventions. ${ }^{16}$ The Higgins I-squared $\left(\mathrm{I}^{2}\right.$ and the Cochran's Q test $(\mathrm{Ph}(\mathrm{Q}))$ tests were performed to assess the statistical heterogeneity of the meta-analysis. A Ph $(\mathrm{Q})$ test with a $\mathrm{p}$ value of less than 0.1 and $\mathrm{I}^{2}$ greater than $50 \%$ indicates significant heterogeneity. The combined OR and HR, together with their 95\% CIs were calculated using the DerSimonian-Laird random-effects model for a $\mathrm{P}_{\text {heterogeneity }}<0.10$ or $\mathrm{I}^{2}>50 \%$. For all other studies, the Mantel-Haenszel fixed effect model was adopted. Studies with inconsistent results were further analysed in a subgroup analysis based on published time, sample size, geographic location, cut-off value and tumour subtype. Meta-regression analysis was used to evaluate the effects of the different subgroups and moderator variables in the meta-analysis. Sensitivity analysis was performed by omitting each single study to evaluate the stability of the results. Begg's tests and funnel plot were applied to evaluate publication biases. All p values were two sided. Statistical significance was considered if the $\mathrm{p}$ value was below 


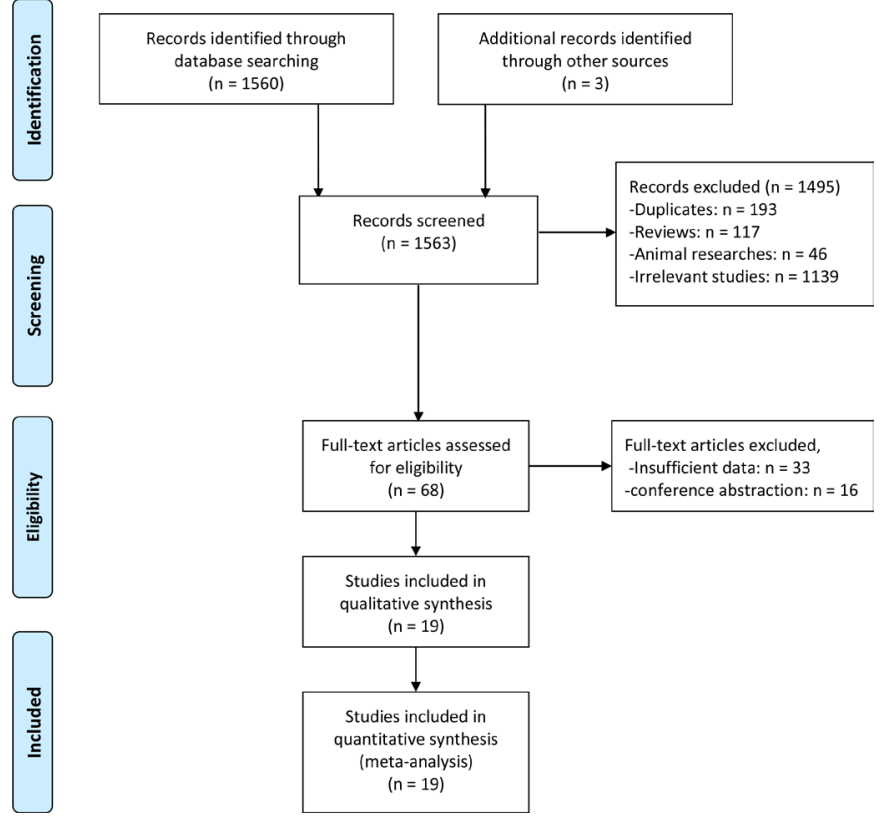

Figure 1 Flow chart of the included studies.

0.05. All data analyses were conducted using Stata V.12.0 (StataCorp) software.

\section{RESULTS}

\section{Overview of studies included in the meta-analysis}

During the preliminary search, a total of 1563 research articles were retrieved. Following evaluation by two independent researchers, nineteen studies with a total of 5910 patients published between 2014 and 2020 were finally included, as illustrated in figure $1 .{ }^{17-35}$ Most studies were conducted in Asia, with $42 \%, 21 \%$ and $11 \%$ of the studies being conducted in China, Korea and Japan, respectively. The rest of the studies were conducted in non-Asian countries, with $10 \%$ being conducted in Spain and the rest in Italy, Mexico, and Turkey. Seventeen studies with 5504 patients analysed the association between the NLR and pCR, 11 studies with 4001 patients reported the relationship between the NLR and DFS, while 6 studies with 3547 patients reported the correlation between NLR and OS in patients with breast cancer treated with NACT. Nine studies enrolled more than 200 patients. The NLR threshold indicative of pCR and DFS varied between studies and ranged from 1.63 to 3.33. Eleven studies had anNLR cut-off value smaller than or equal to 2.3, and seven studies reported an NLR greater than 2.3. Eighteen studies involved early-stage and locally advanced disease stages (I-III/II-III), and only one study included advanced-stage disease (II-IV). Two of the studies included the hormone receptor (HR) positive and the human epidermal growth factor receptor 2 (HER2) negative (HR+/HER2-) breast cancer subtype. Four studies only included the triple-negative breast cancer (TNBC) subtype, one study included the HER2positive breast cancer subtype only, and another study examined both the HR+/HER2- and the TNBC subtypes.
The other studies included all breast cancer subtypes. In almost all studies, the patients were treated with the standard anthracycline and/or taxane NACT. However, two studies included other NACT combinations based on everolimus, platinum compounds and vinorelbine. Neoadjuvant trastuzumab was administered to patients whose tumours were HER2-positive. The study characteristics are summarised in table 1.

\section{Association between the NLR and PCR}

A link between a higher NLR and negative pCR was identified in seventeen studies (OR 1.620, 95\% CI 1.209 to $2.169, \mathrm{I}^{2}=66.0 \%, \mathrm{p}<0.001$, random effects model; figure 2A). Following stratification by publication year, we found a pooled OR of 1.452 (95\% CI 1.133 to 1.860 ; $\mathrm{p}=0.003$ ) for studies published after 2018. Conversely, we did not identify a statistically significant difference in the OR (OR 2.144; 95\% CI 0.949 to 4.844; $\mathrm{p}=0.067$ ) and subgroup analysis $(\mathrm{p}=0.571)$ for studies published before 2018 (table 2). The data were then stratified into Asian and non-Asian. A statistically significant relationship between NLR and pCR was identified in Asian studies (OR 1.726; $95 \%$ CI 1.167 to 2.553; $\mathrm{p}=0.006$ ), but the same finding was not observed in non-Asian studies (OR 1.189; 95\% CI 0.974 to $1.451 ; \mathrm{p}=0.089$ ) (table 2 ).

Further analysis was also conducted to evaluate the impact of sample size on the study outcomes. The ORs were 1.254 (95\% CI 0.970 to 1.621 ) for studies with a sample size greater than 200 and 2.783 (95\% CI 1.484 to 5.221) for studies with a sample size below 200. The OR for high NLR in studies with a sample size below 200 was significantly higher when compared with the OR in studies with a sample size above 200 ( $\mathrm{p}$ for subgroup difference=0.04) (table 2 ). When evaluating the NLR cutoff values, an NLR below or equal to 2.3 did not show a statistically significant association with pCR (OR 1.387; $95 \%$ CI 0.930 to $2.069 ; p=0.108$ ), while a statistically significant association was noted for NLR values greater than 2.3 (OR 2.334; 95\% CI 1.345 to 4.051; p=0.003). Further, subgroup analysis was performed by tumour subtypes. In the TNBC subtype a statistical significance was noted between NLR and pCR (OR 2.905; 95\% CI 1.350 to 6.249; $\mathrm{p}=0.006)$. The ORs were 1.491 (95\% CI 1.040 to 2.137$)$ for all breast cancer subtypes and 1.320 (95\% CI 0.646 to 2.697) for HR+/ HER2-. Differences between tumour subtypes were not statistically significant ( $p$ for subgroup difference $=0.256) \quad($ table 2$)$.

\section{Association between NLR and breast cancer survival}

Eleven studies with 4001 cases were identified comparing high pretreatment NLR and DFS in patients with breast cancer. Since the $\mathrm{I}^{2}$ value indicated significant heterogeneity $\left(\mathrm{I}^{2}=71.30 \%, \mathrm{Ph}=0.000\right)$, a random effects model was applied (figure 2B). The pooled results of these studies demonstrated a statistically significant correlation between high pretreatment NLR levels and DFS with an HR of 2.269 (95\% CI 1.557 to 3.307, p<0.001, table 3). Subgroup analyses by publication year using fixed-effects 


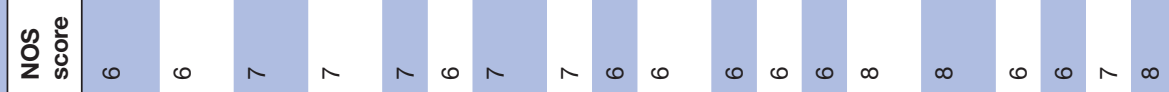

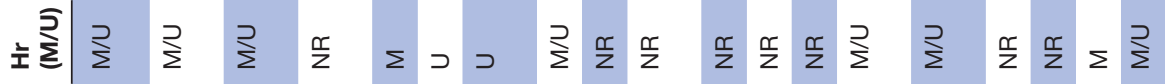

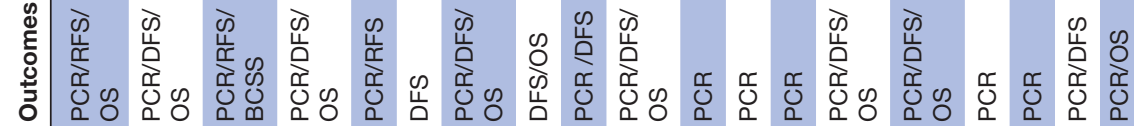
๖े

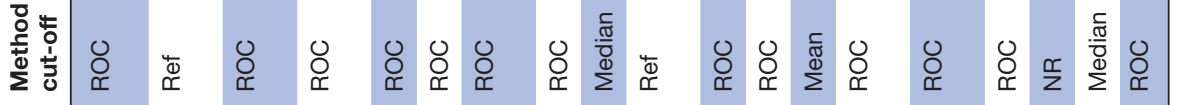

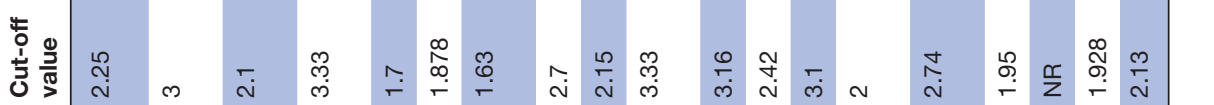

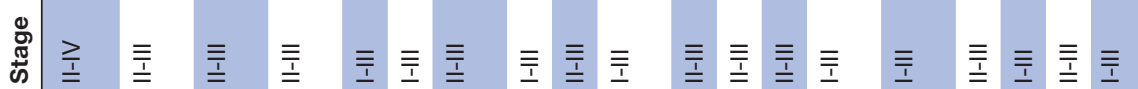

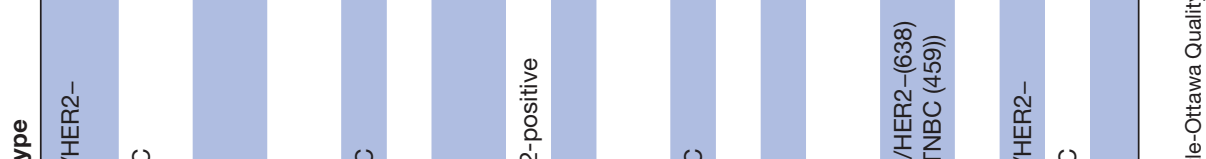

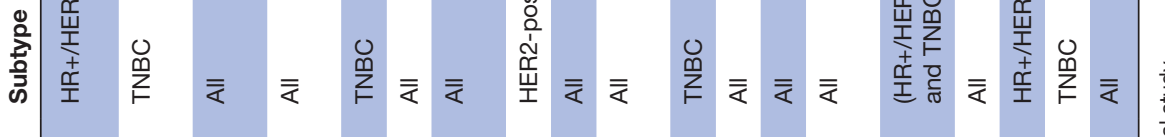

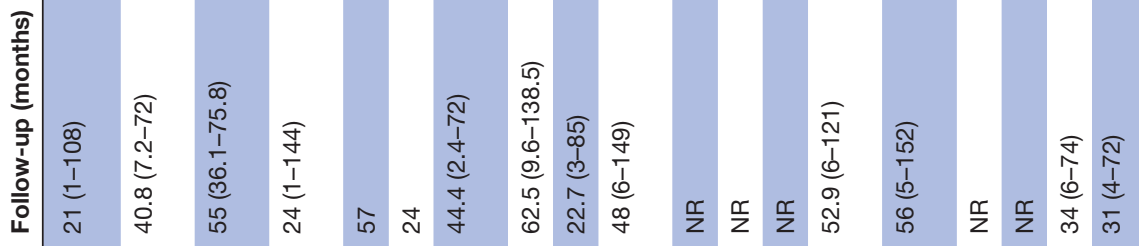

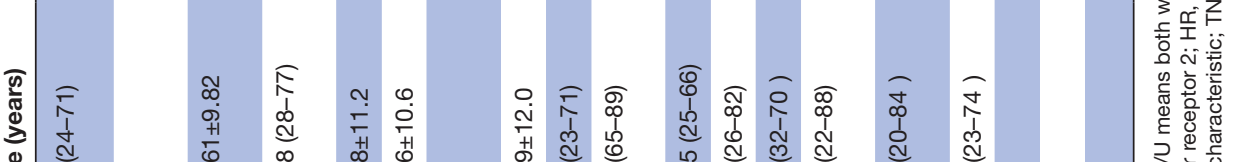

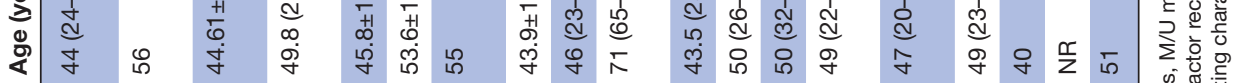

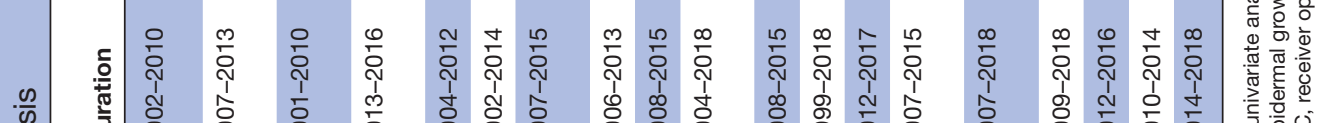

命

כل

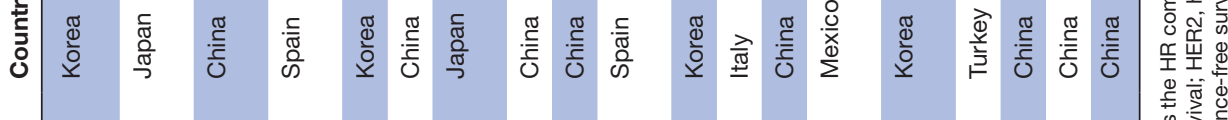

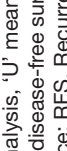

๓ॉ

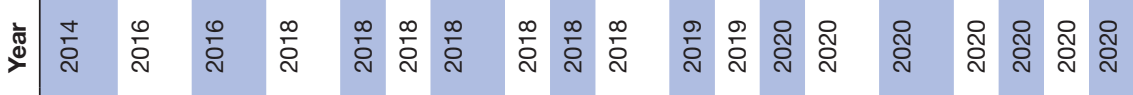

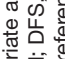

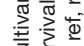

है.

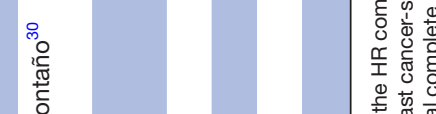

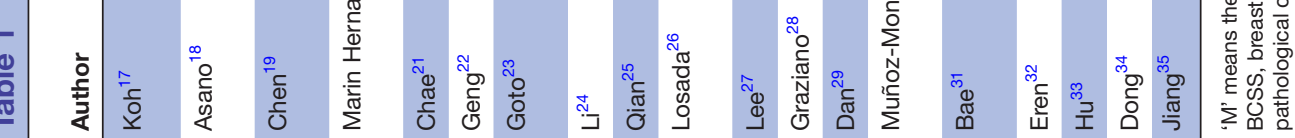




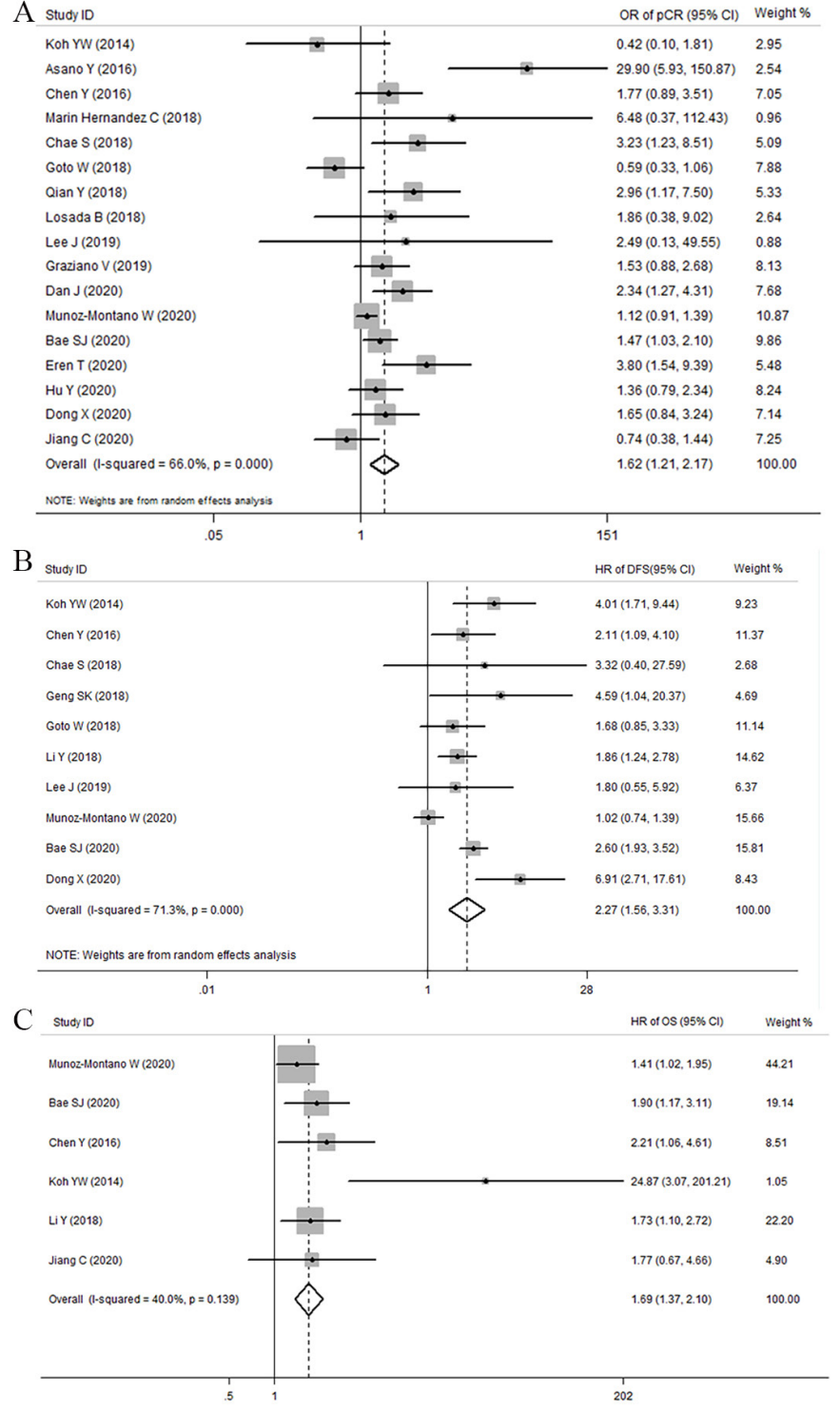

Figure 2 Forest plots illustrating the association between NLR and PCR (A), DFS (B) and OS (C) of breast cancer treated with NAC. Results are presented as individual and pooled or or HR with their $95 \% \mathrm{Cl}$. DFS, disease-free survival; NAC, neoadjuvant chemotherapy; NLR, neutrophilto-lymphocyte ratio; OS, overall survival; PCR, pathological complete response.

model revealed a pooled HR of 2.120 (95\% CI 1.599 to $2.811, \mathrm{p}<0.001$ ) and 2.261 (95\% CI 1.065 to 4.801 , $\mathrm{p}=0.034$ ) for studies published before and after 2018, respectively. Stratification by geographic location showed that NLR was not associated with DFS in non-Asian populations $(\mathrm{p}=0.912)$. Conversely, in Asian patients, an elevated NLR was linked with shorter DFS (HR obtained from fixed-effects model: 2.478, 95\% CI 1.914 to 3.208, $\left.\mathrm{p}<0.001, \quad \mathrm{I}^{2}=22.70 \%, \quad \mathrm{Ph}=0.241\right)$. Subgroup analyses by sample size revealed a pooled HR of 4.091 (95\% CI 2.464 to $6.793, \mathrm{p}<0.001, \mathrm{I}^{2}=0.00, \mathrm{Ph}=0.542$ ) for sample size smaller than 200 and 2.604 (95\% CI 1.927 to 3.518, $\mathrm{p}=0.007$ ) for sample size greater than 200. In addition, subgroup analysis was performed according to a cut-off value of 2.3, tumour subtype, and univariate and multivariate analysis (table 3 ). The heterogeneity issue in the analysed study was addressed by performing meta-regression analyses based on publication year (before 2018 or after 2018 ), location (Asian or non-Asian), sample size $(\leq 200$ or $>200)$, cut-off value $(\leq 2.3$ or $>2.3)$, tumour subtype, and univariate analysis and multivariate analysis. The meta-regression analyses did account for the heterogeneity source caused by study location and sample size ( $p$ for subgroup difference $=0.008$ and 0.044 , respectively.) (table 3). The fixed-effects model $\left(\mathrm{I}^{2}=40.00 \%, \mathrm{Ph}=0.139\right.$; figure 2C) was also used for studies evaluating OS. The results revealed that an elevated NLR predicted a worse OS with a combined HR of 1.691 (95\% CI 1.365 to 2.096, $\mathrm{p}=0.000$; table 3 ). We performed further subgroup analysis for the OS subset using various clinical features included in the heterogeneity literature, and no significant changes were identified after stratification (online supplemental table 1 ).

\section{Sensitivity analysis}

According to the sensitivity analysis, the majority of the studies were near the central line with no clear deviation, as illustrated in figure $3 \mathrm{~A}, \mathrm{~B}$.

\section{Publication biases}

Visual inspection of the Begg's test or funnel plots did not reveal any publication bias (figure $4 \mathrm{~A}, \mathrm{~B}$ ). The Begg's test z-score $\mathrm{p}$ values were 0.108 and 0.474 for $\mathrm{pCR}$ and DFS, respectively.

\section{DISCUSSION}

This meta-analysis assessed the impact of pretreatment NLR on pCR and survival on 5504 patients with breast cancer from 17 studies. The findings indicate that elevated NLR significantly predicted poor pCR in patients with breast cancer with NACT (OR 1.620, 95\% CI 1.209 to $2.169, \mathrm{p}<0.001)$. Only 11 studies analysed the relationship between NLR elevation and DFS in patients with breast cancer. The combined HR of 2.269 based on the randomeffects model (95\% CI: 1.557 to $3.307, \mathrm{I}^{2}=71.30 \%$, $\mathrm{Ph}=0.000, \mathrm{p}<0.001)$ demonstrated that patients with an elevated NLR had worse DFS postsurgery. Furthermore, NLR elevation was also associated with worse OS (HR $1.691,95 \%$ CI 1.365 to $2.096, \mathrm{p}<0.001$ ) in 3547 patients with breast cancer in six studies. ${ }^{17} 1924303135$ Consistent with previously published studies, our findings suggest that pCR was more likely to be achieved in patients with breast cancer with an NLR below 2.3, leading to improved DFS and OS irrespective of the clinical stage, nuclear grade, the value of Ki67 expression levels and chemotherapy regimen. ${ }^{2418}$

Following subgroup analysis, we found that NLR was only significantly associated with pCR in patients with TNBC, ${ }^{18} 21273134$ but not in those who were HR+/ HER2-. ${ }^{17} 33$ NLR tends to be relatively low in TNBC subtypes partially due to high lymphocyte activity. ${ }^{27}$ 
Table 2 Association between NLR and pCR for different subgroups

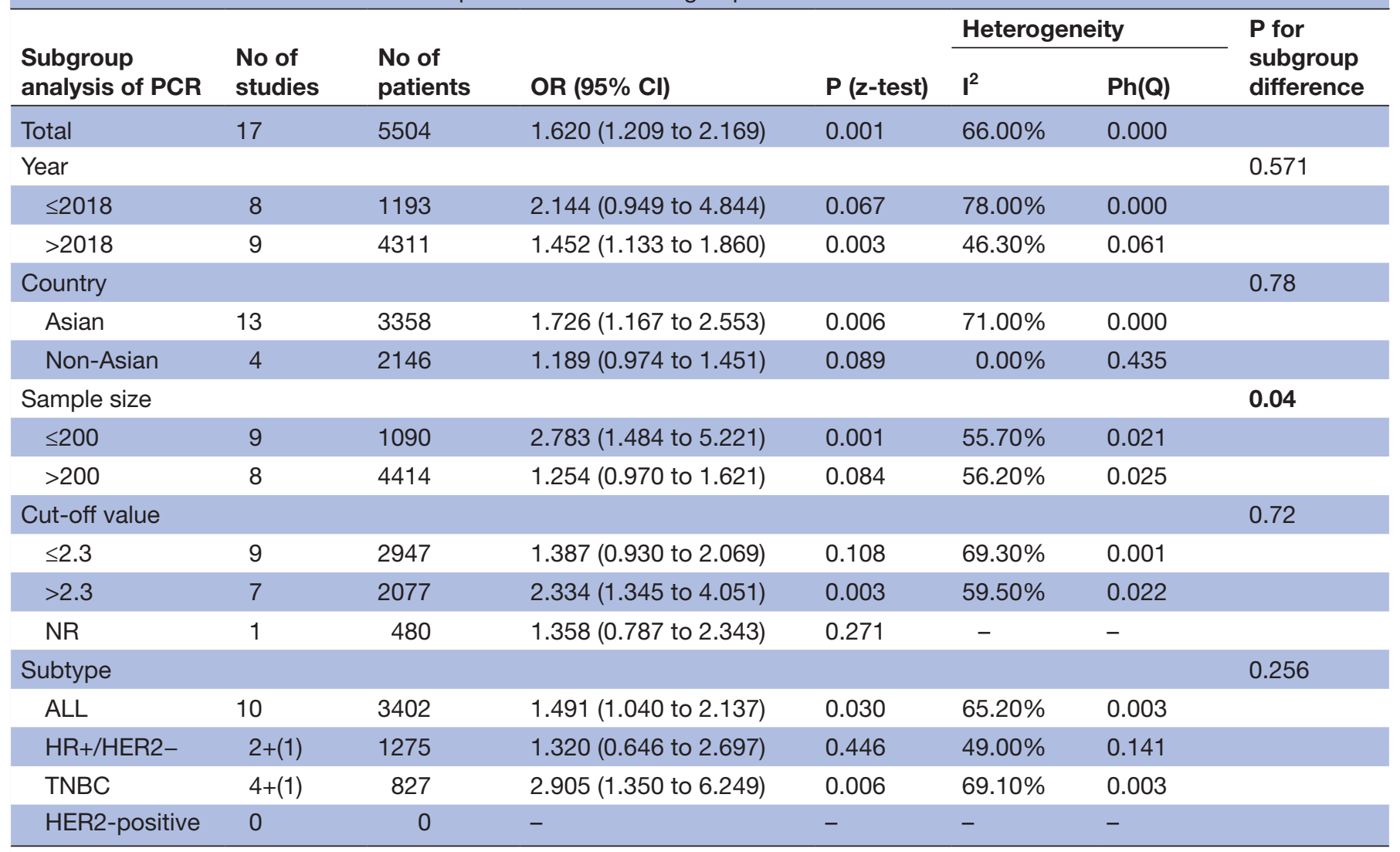

Bold values mean $\mathrm{p}<0.05$.

HER2, human epidermal growth factor receptor 2; HR, hormone receptor; NLR, neutrophil-to-lymphocyte ratio; NR, not reported; pCR, pathological complete response; $\mathrm{Ph}(\mathrm{Q})$, $\mathrm{p}$ values of $\mathrm{Q}$ test for heterogeneity test; TNBC, triple-negative breast cancer.

The pCR rate has a unique prognostic value in TNBC subtype. $^{36} 37$ In patients with low NLR, chemotherapy stimulates the immune response, thereby accelerating the antitumour effect, ${ }^{38}$ indicating that NLR could potentially be used as an immunoinflammatory marker to predict the response to NACT in TNBC patients.

We also evaluated the relationship between NLR and prognosis. As opposed to the result by Xue et al and Cullinane $e t a l^{39}{ }^{40}$ the pooled data indicated that high NLR was significantly correlated with OS $(\mathrm{p}=0.001)$ and DFS $(p<0.001)$. Furthermore, analysis based on tumour subtype revealed a statistically significant correlation between NLR and DFS in HR+/HER2- and TNBC subtypes. Koh et al reported the NLR in 157 patients with breast cancer and also found a statistically significant relationship among NLR elevation, DFS and OS in patients with HR+/HER2-. ${ }^{17}$ Similarly, Bae et al evaluated 638 HR+/HER2- patients with breast cancer and also identified that a high NLR as a significant independent parameter affecting DFS and OS. ${ }^{31}$ The TNBC subtype of 827 patients was studied by four centres and was significantly linked with a worse prognosis in all studies. ${ }^{18} 213134$ This finding is consistent with previous studies confirming that TNBC was more immunogenic than other subtypes of breast cancer, and higher pretreatment levels of tumourinfiltrating lymphocytes were correlated with improved survival in TNBC patients treated with NACT. ${ }^{41-43}$ Nevertheless, our study included a larger number of patients and studies conducted after 2018. These studies showed a more significant correlation with survival, possibly due to the provision of a more standardised preoperative treatment.

The underlying mechanisms behind the role of elevated NLR levels and poor outcomes in cancer patients are poorly understood. A possible explanation could be that NLR levels are associated with inflammation. Studies have shown that inflammation caused by the tumour microenvironment promotes the initiation, proliferation, haematogenous spread and survival of tumour cells. This eventually reduces the response to anticancer agents. ${ }^{67}$ Neutrophils form part of the tumour microenvironment and therefore have an important role in tumour progression and in the development of pathways leading to treatment resistance. ${ }^{44}$ It has been suggested that the tumour-induced neutrophils suppress cytotoxic CD8 + T lymphocytes by producing inducible nitric oxide synthase, a major inflammatory mediator, limiting tumour spread. ${ }^{45}$ This implies that the pretreatment immunityinflammation peripheral blood index measured in the form of a combined neutrophil and lymphocyte counts is more likely to represent a better response to therapy. 


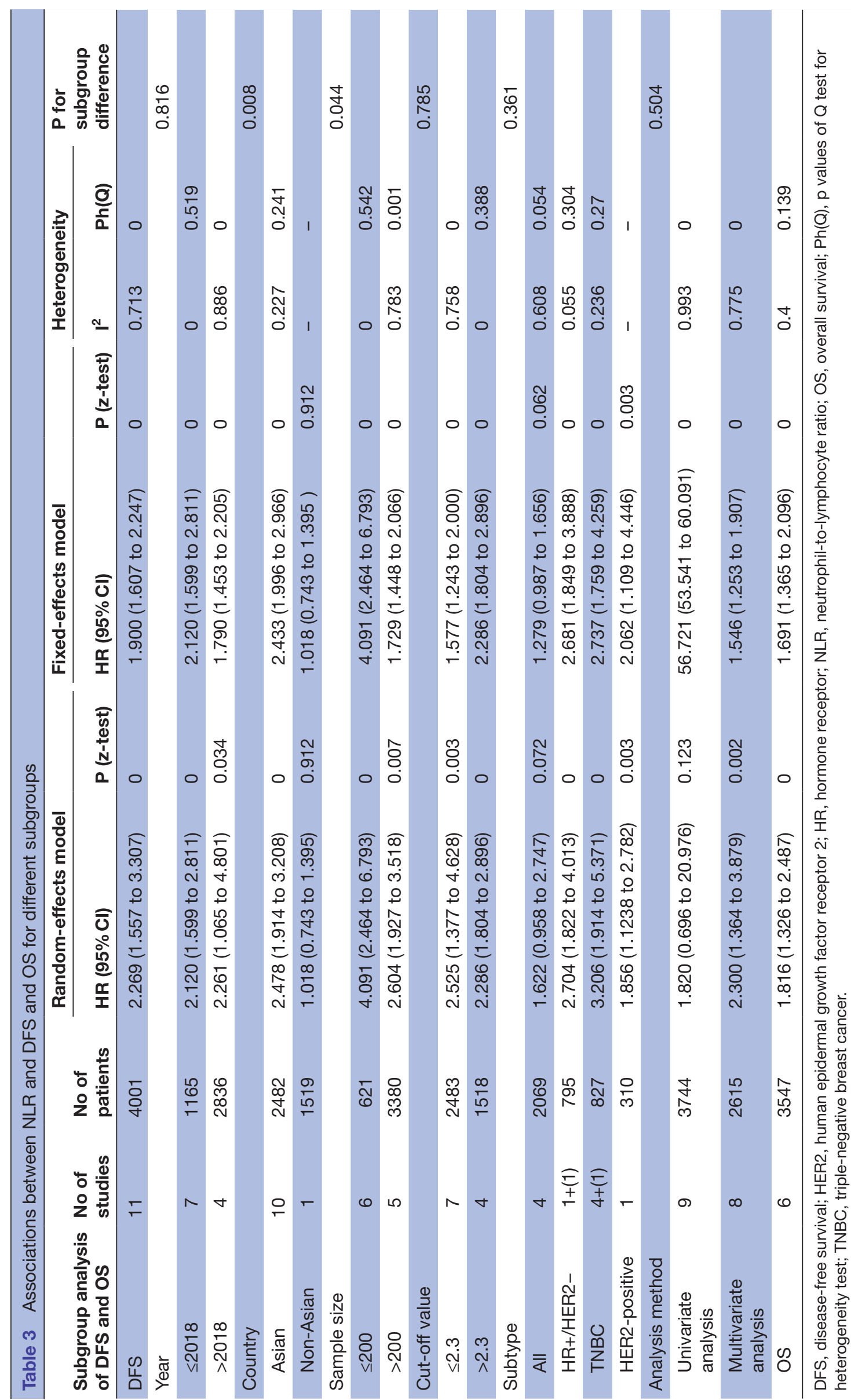

\begin{tabular}{l}
0 \\
3 \\
\hdashline \\
0 \\
0 \\
0
\end{tabular} 

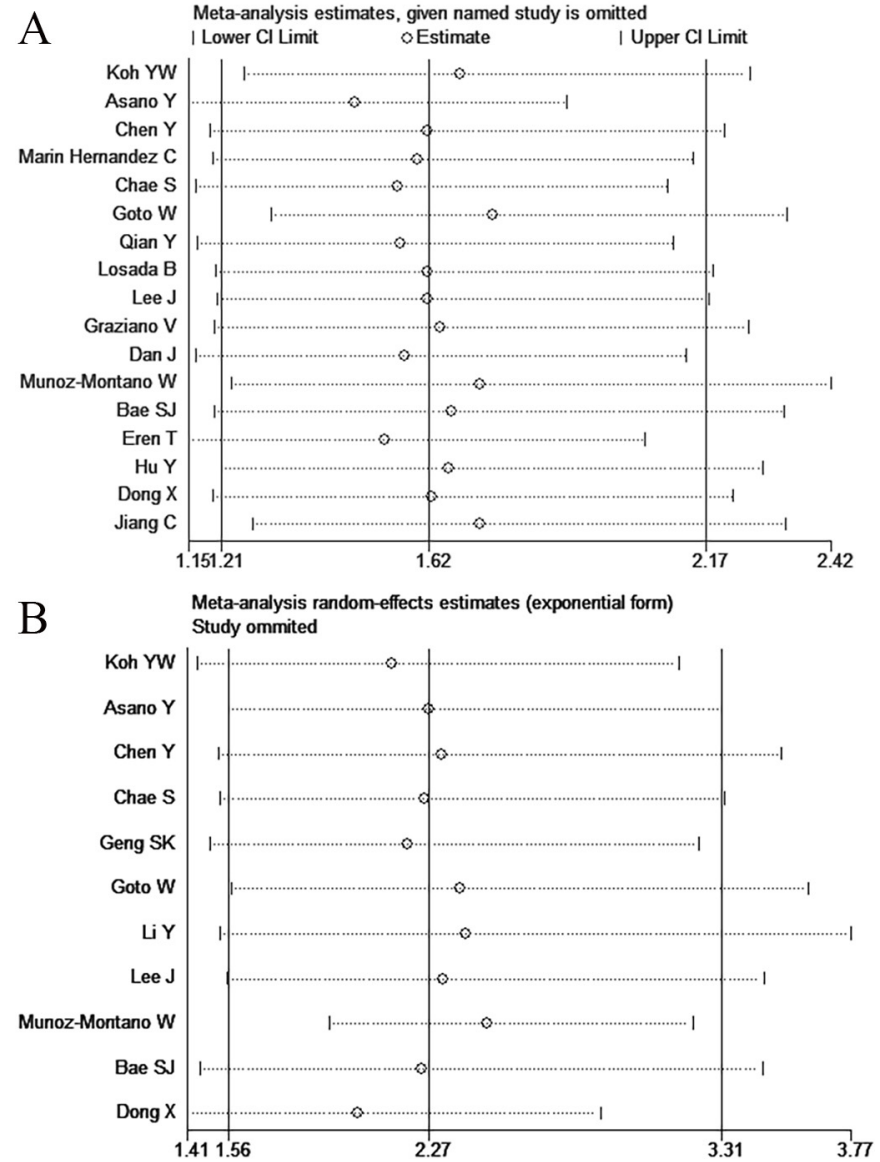

Figure 3 Sensitivity analysis of the relationships between high NLR and PCR (A) and DFS (B). DFS, disease-free survival; NLR, neutrophil-to-lymphocyte ratio; PCR, pathological complete response.

This study has several limitations that need to be considered. First of all, the limited availability of HER2positive breast cancer data could potentially have biased the results of the research findings. Second, the studies included in our analysis were all retrospective. In addition, the NACT regimens and doses could have varied among studies. Almost all patients received standardised NACT based on anthracycline and/or taxane. Therefore, the impact of using different NACT regimens could not be evaluated in our meta-analysis. Besides, the tendency of studies with positive results to be published and the fact that we could only include written English papers might have introduced potential publication bias in our meta-analysis, although the funnel plots and Begg's test indicated that there were no significant publication bias in pCR and DFS studies $(p>0.05)$. Finally, the patient characteristics, including age distribution, presence of concurrent disease, geographic location and NLR cutoff value varied among studies, potentially leading to heterogeneity, which is a common limitation of all metaanalyses. The need to pool the HR for OS results could have increased the heterogeneity further. Since it was not possible to account for all sources of heterogeneity in this meta-analysis, further research is recommended to assess
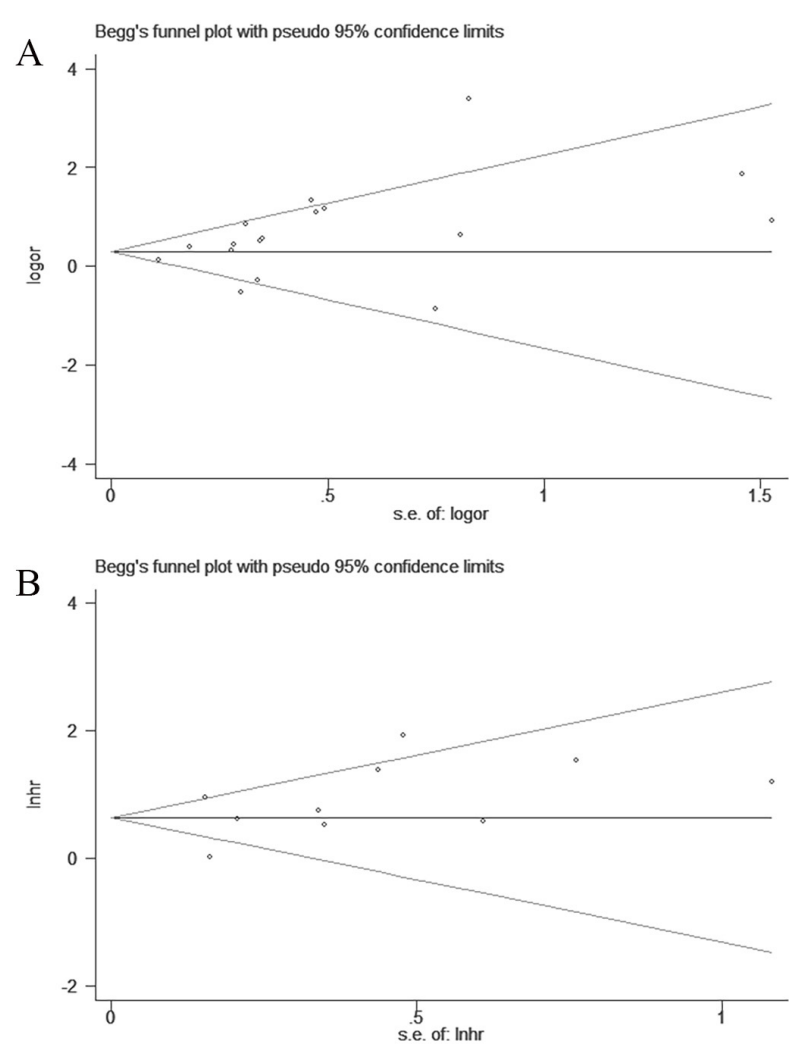

Figure 4 Funnel plots for the detection of publication bias of the PCR (A) and DFS (B). DFS, disease-free survival; PCR, pathological complete response.

the relationship between NLR and tumour prognosis more accurately.

\section{CONCLUSION}

This study found a reduced association between high levels of NLR and pCR achievement following NACT in patients with HER2-negative breast cancer. High values of NLR seemed also to be associated with worse survival. This implies that NLR is a promising non-invasive prognostic inflammation marker that could be developed to assist the clinical decision-making process regarding the use of NACT in patients with breast cancer. However, further studies with larger samples are recommended to explain the relationship between high NLR and worse survival for all breast cancer subtypes.

Contributors QZ and XH designed the study. QZ, JD, QS, NL, YP and XH collected the data. QZ drafted the manuscript. QZ and XH contributed to the interpretation of the results and critical revision of the manuscript for important intellectual content and approved the final version of the manuscript. All authors read and approved the final manuscript.

Funding This work was partially supported by Anhui Provincial Key Research and Development Programme Science and Technology Support Key Projects (No. 1804h08020259) and Anhui Province Natural Science Foundation (No.1908085MH286).

Competing interests None declared.

Patient consent for publication Not applicable.

Provenance and peer review Not commissioned; externally peer reviewed.

Data availability statement Data are available in a public, open access repository. 
Supplemental material This content has been supplied by the author(s). It has not been vetted by BMJ Publishing Group Limited (BMJ) and may not have been peer-reviewed. Any opinions or recommendations discussed are solely those of the author(s) and are not endorsed by BMJ. BMJ disclaims all liability and responsibility arising from any reliance placed on the content. Where the content includes any translated material, BMJ does not warrant the accuracy and reliability of the translations (including but not limited to local regulations, clinical guidelines, terminology, drug names and drug dosages), and is not responsible for any error and/or omissions arising from translation and adaptation or otherwise.

Open access This is an open access article distributed in accordance with the Creative Commons Attribution Non Commercial (CC BY-NC 4.0) license, which permits others to distribute, remix, adapt, build upon this work non-commercially, and license their derivative works on different terms, provided the original work is properly cited, appropriate credit is given, any changes made indicated, and the use is non-commercial. See: http://creativecommons.org/licenses/by-nc/4.0/.

\section{ORCID iD}

Qiong Zhou http://orcid.org/0000-0002-8334-5527

\section{REFERENCES}

1 Siegel RL, Miller KD, Jemal A. Cancer statistics, 2020. CA Cancer J Clin 2020;70:7-30.

2 Prowell TM, Pazdur R. Pathological complete response and accelerated drug approval in early breast cancer. N Engl J Med 2012;366:2438-41.

3 Huang M, O'Shaughnessy J, Zhao J, et al. Association of pathologic complete response with long-term survival outcomes in triple-negative breast cancer: a meta-analysis. Cancer Res 2020;80:5427-34.

4 Xu W, Chen X, Deng F, et al. Predictors of neoadjuvant chemotherapy response in breast cancer: a review. Onco Targets Ther 2020;13:5887-99.

5 Mantovani A, Allavena P, Sica A, et al. Cancer-Related inflammation. Nature 2008;454:436-44.

6 Hanahan D, Weinberg RA. Hallmarks of cancer: the next generation. Cell 2011;144:646-74.

7 Diakos Cl, Charles KA, McMillan DC, et al. Cancer-related inflammation and treatment effectiveness. Lancet Oncol 2014;15:e493-503.

8 Dolan RD, McMillan DC. The prevalence of cancer associated systemic inflammation: implications of prognostic studies using the Glasgow prognostic score. Crit Rev Oncol Hematol 2020;150:102962.

9 Li B, Zhou P, Liu Y, et al. Platelet-To-Lymphocyte ratio in advanced cancer: review and meta-analysis. Clin Chim Acta 2018;483:48-56.

10 Allin $\mathrm{KH}$, Nordestgaard BG. Elevated C-reactive protein in the diagnosis, prognosis, and cause of cancer. Crit Rev Clin Lab Sci 2011:48:155-70.

11 Schettini F, Sobhani N, lanza A, et al. Immune system and angiogenesis-related potential surrogate biomarkers of response to everolimus-based treatment in hormone receptor-positive breast cancer: an exploratory study. Breast Cancer Res Treat 2020;184:421-31.

12 Templeton AJ, McNamara MG, Šeruga B, et al. Prognostic role of neutrophil-to-lymphocyte ratio in solid tumors: a systematic review and meta-analysis. J Natl Cancer Inst 2014;106:dju124.

$13 \mathrm{Li} \mathrm{X,} \mathrm{Dai} \mathrm{D,} \mathrm{Chen} \mathrm{B,} \mathrm{et} \mathrm{al.} \mathrm{The} \mathrm{value} \mathrm{of} \mathrm{neutrophil-to-lymphocyte} \mathrm{ratio}$ for response and prognostic effect of neoadjuvant chemotherapy in solid tumors: a systematic review and meta-analysis. $J$ Cancer 2018;9:861-71.

14 Oremus M, Oremus C, Hall GBC, et al. Inter-Rater and test-retest reliability of quality assessments by novice student raters using the Jadad and Newcastle-Ottawa scales. BMJ Open 2012;2:e001368.

15 Parmar MK, Torri V, Stewart L. Extracting summary statistics to perform meta-analyses of the published literature for survival endpoints. Stat Med 1998;17:2815-34.

16 Higgins JPT, Thomas J, Chandler J. Cochrane Handbook for systematic reviews of interventions version 6.2 (updated February 2021), 2021. Available: www.training.cochrane.org/handbook

17 Koh YW, Lee HJ, Ahn J-H, et al. Prognostic significance of the ratio of absolute neutrophil to lymphocyte counts for breast cancer patients with ER/PR-positivity and HER2-negativity in neoadjuvant setting. Tumour Biol 2014;35:9823-30.

18 Asano Y, Kashiwagi S, Onoda N, et al. Predictive value of neutrophil/ lymphocyte ratio for efficacy of preoperative chemotherapy in triplenegative breast cancer. Ann Surg Oncol 2016;23:1104-10.
19 Chen Y, Chen K, Xiao X, et al. Pretreatment neutrophil-to-lymphocyte ratio is correlated with response to neoadjuvant chemotherapy as an independent prognostic indicator in breast cancer patients: a retrospective study. BMC Cancer 2016;16:320.

20 Marín Hernández C, Piñero Madrona A, Gil Vázquez PJ, et al. Usefulness of lymphocyte-to-monocyte, neutrophil-to-monocyte and neutrophil-to-lymphocyte ratios as prognostic markers in breast cancer patients treated with neoadjuvant chemotherapy. Clin Trans/ Oncol 2018;20:476-83.

21 Chae S, Kang KM, Kim HJ, et al. Neutrophil-Lymphocyte ratio predicts response to chemotherapy in triple-negative breast cancer. Curr Oncol 2018;25:113-9.

22 Geng S-K, Fu S-M, Fu Y-P, et al. Neutrophil to lymphocyte ratio is a prognostic factor for disease free survival in patients with breast cancer underwent curative resection. Medicine 2018;97:e11898.

23 Goto W, Kashiwagi S, Asano Y, et al. Predictive value of lymphocyteto-monocyte ratio in the preoperative setting for progression of patients with breast cancer. BMC Cancer 2018;18:1137.

$24 \mathrm{Li} \mathrm{Y,} \mathrm{Shao} \mathrm{Y,} \mathrm{Bai} \mathrm{L,} \mathrm{et} \mathrm{al.} \mathrm{Increased} \mathrm{derived} \mathrm{neutrophil-to-lymphocyte}$ ratio and breast Imaging-Reporting and data system classification predict poor survival in patients with non-distant metastatic HER2+ breast cancer treated with neoadjuvant chemotherapy. Cancer Manag Res 2018;10:3841-7.

25 Qian Y, Tao J, Li X, et al. Peripheral inflammation/immune indicators of chemosensitivity and prognosis in breast cancer patients treated with neoadjuvant chemotherapy. Onco Targets Ther 2018;11:1423-32.

26 Losada B, Guerra JA, Malón D, et al. Pretreatment neutrophil/ lymphocyte, platelet/lymphocyte, lymphocyte/monocyte, and neutrophil/monocyte ratios and outcome in elderly breast cancer patients. Clin Transl Oncol 2019;21:855-63.

27 Lee J, Kim D-M, Lee A. Prognostic role and clinical association of tumor-infiltrating lymphocyte, programmed death ligand-1 expression with neutrophil-lymphocyte ratio in locally advanced triple-negative breast cancer. Cancer Res Treat 2019;51:649-63.

28 Graziano V, Grassadonia A, lezzi L, et al. Combination of peripheral neutrophil-to-lymphocyte ratio and platelet-tolymphocyte ratio is predictive of pathological complete response after neoadjuvant chemotherapy in breast cancer patients. Breast 2019;44:33-8.

29 Dan J, Tan J, Huang J, et al. The dynamic change of neutrophil to lymphocyte ratio is predictive of pathological complete response after neoadjuvant chemotherapy in breast cancer patients. Breast Cancer 2020;27:982-8.

30 Muñoz-Montaño W, Cabrera-Galeana P, Alvarado-Miranda A, et al. Prognostic value of the pretreatment neutrophil-to-lymphocyte ratio in different phenotypes of locally advanced breast cancer during neoadjuvant systemic treatment. Clin Breast Cancer 2020;20:307-16.

31 Bae SJ, Cha YJ, Yoon C, et al. Prognostic value of neutrophilto-lymphocyte ratio in human epidermal growth factor receptor 2-negative breast cancer patients who received neoadjuvant chemotherapy. Sci Rep 2020;10:13078.

32 Eren T, Karacin C, Ucar G, et al. Correlation between peripheral blood inflammatory indicators and pathologic complete response to neoadjuvant chemotherapy in locally advanced breast cancer patients. Medicine 2020;99:e20346.

33 Hu Y, Wang S, Ding N, et al. Platelet/Lymphocyte Ratio Is Superior to Neutrophil/Lymphocyte Ratio as a Predictor of Chemotherapy Response and Disease-free Survival in Luminal B'like (HER2') Breast Cancer. Clin Breast Cancer 2020;20:e403-9.

34 Dong X, Liu C, Yuan J, et al. Prognostic roles of neutrophil-tolymphocyte ratio and stromal tumor-infiltrating lymphocytes and their relationship in locally advanced triple-negative breast cancer treated with neoadjuvant chemotherapy. Breast Care 2021;16:328-34.

35 Jiang C, Lu Y, Zhang S, et al. Systemic immune-inflammation index is superior to neutrophil to lymphocyte ratio in prognostic assessment of breast cancer patients undergoing neoadjuvant chemotherapy. Biomed Res Int 2020;2020:1-10.

36 Masuda H, Baggerly KA, Wang Y, et al. Differential response to neoadjuvant chemotherapy among 7 triple-negative breast cancer molecular subtypes. Clin Cancer Res 2013;19:5533-40.

37 Denkert C, von Minckwitz G, Darb-Esfahani S, et al. Tumourinfiltrating lymphocytes and prognosis in different subtypes of breast cancer: a pooled analysis of 3771 patients treated with neoadjuvant therapy. Lancet Oncol 2018;19:40-50.

38 Stanton SE, Disis ML. Clinical significance of tumor-infiltrating lymphocytes in breast cancer. J Immunother Cancer 2016;4:59.

39 Xue LB, Liu YH, Zhang B, et al. Prognostic role of high neutrophilto-lymphocyte ratio in breast cancer patients receiving neoadjuvant chemotherapy: meta-analysis. Medicine 2019;98:e13842. 
40 Cullinane C, Creavin B, O'Leary DP, et al. Can the neutrophil to lymphocyte ratio predict complete pathologic response to neoadjuvant breast cancer treatment? A systematic review and meta-analysis. Clin Breast Cancer 2020;20:e675-81.

41 Loi S, Drubay D, Adams S, et al. Tumor-Infiltrating lymphocytes and prognosis: a pooled individual patient analysis of early-stage triplenegative breast cancers. J Clin Oncol 2019;37:559-69.

42 Gruosso T, Gigoux M, Manem VSK, et al. Spatially distinct tumor immune microenvironments stratify triple-negative breast cancers. $J$ Clin Invest 2019;129:1785-800.
43 Telli ML, Chu C, Badve SS, et al. Association of Tumor-Infiltrating Lymphocytes with Homologous Recombination Deficiency and BRCA1/2 Status in Patients with Early Triple-Negative Breast Cancer: A Pooled Analysis. Clin Cancer Res 2020;26:2704-10.

44 Carnevale S, Ghasemi S, Rigatelli A, et al. The complexity of neutrophils in health and disease: focus on cancer. Semin Immunol 2020;48:101409.

45 Coffelt SB, Kersten K, Doornebal CW, et al. II-17-Producing $\gamma \delta \mathrm{T}$ cells and neutrophils conspire to promote breast cancer metastasis. Nature 2015;522:345-8. 